\title{
CONFLITOS DE AGÊNCIA: UM ESTUDO COMPARATIVO DOS ASPECTOS INERENTES A EMPRESAS TRADICIONAIS E COOPERATIVAS DE CRÉDITO
}

\section{CONFLICTS OF AGENCY: A COMPARATIVE ST UDY OF ISSUES ATT ACHED TO S AND T RADIT IONALCOMPANIES AND CREDIT COOPERATIVE}

ROMEU EUGÊNIO DE LIMA

Banco Central do Brasil

E-mail: romeu.lima@bcb.gov.br

\author{
MARCELO BICALHO VITURINO DE ARAÚJ O \\ Banco Central do Brasil \\ E-mail: bicalho.araujo@bcb.gov.br
}

\author{
HUDSON FERNANDES AMARAL \\ Doutor em Sciences de Gestion pela \\ Université Pierre Mendés France - Grenoble II (França) \\ Professor Associado da Faculdade de Ciências \\ Econômicas da Universidade Federal de Minas Gerais \\ E-mail: hfamaral@face.ufmg.br
}

\section{Resumo}

0 presente artigo procura analisar e comparar, com base em referências bibliográficas, os potenciais conflitos de agência em dois diferentes tipos de organizações, a saber: empresas tradicionais e cooperativas de crédito. Tal comparação se justifica pelo fato das cooperativas de crédito serem instituições financeiras menos estudadas em relação às instituições tradicionais, apesar de atualmente participarem da política de democratização e acesso ao crédito do Governo Federal, com recentes avanços normativos. Além disso, os conflitos de agência e sua minimização vêm recebendo destaque crescente como instrumentos de viabilidade e redução de riscos. Observou-se que existem conflitos de agência diferentes em cooperativas de crédito, quando comparados com as empresas tradicionais. Tal fato pode ser um indicativo da necessidade de aplicação de diferentes soluções de governança para as cooperativas. Entretanto, alguns autores sugerem que os conflitos de agência nas cooperativas de crédito podem ser superados adotando-se providências genéricas, tais como: regras bem definidas, controles internos eficazes, adequação dos serviços prestados, adoção de regulamentações prudenciais e supervisão externa. Essas providências são as sugeridas para as empresas tradicionais, o que, a princípio, poderia ser considerada uma contradição em relação à constatação de que existem conflitos de agência diferentes nas cooperativas de crédito.

Palavras-chave: Cooperativas de crédito, empresas tradicionais, Teoria da Agência, conflitos de agência.

\section{Abstract}

The purpose of this article is, based on the relevant bibliography, to analyze and compare potential agency conflicts in two different kinds of organizations: traditional companies and credit unions. This is justified by the fact that credit unions are the less studied financial institutions, although, nowadays, they play an important role in Brazilian government access credit policies. Furthermore, agency conflicts and their minimization are considered viability and risk reduction instruments. There are different agency conflicts in credit unions compared with the ones observed in traditional companies. This may indicative that different governance solutions are necessary to credit unions. However, some authors suggest that agency conflicts in credit unions can be solved by generic actions, such as: well-defined rules, efficient internal controls, service adequacy, prudential rules adoption and extern supervision. These approaches are the same adopted in traditional companies, what, in principle, would be considered a paradox if we consider that agency conflicts are different in credit unions.

Key-words: Credit unions, traditional companies, Agency Theory, agency conflicts. 


\section{INTRODUÇÃO}

A Teoria da Agência ou Teoria do Agente-Principal foi desenvolvida por J ensen e Meckling (1976) e tem sua raiz no utilitarismo econômico (Ross, 1973). Essa teoria tem como premissa básica a existência de um mercado regido por contratos firmados entre os agentes econômicos, quer sejam empresas, governo ou pessoas físicas. Portanto, toda a atividade econômica reduzir-se-ia a uma série de contratos bilaterais que poderiam ser firmados ou rompidos a qualquer momento por qualquer uma das partes.

0 foco principal dessa teoria está no relacionamento entre agente e principal, no qual o agente dispõe de informações privilegiadas e suas ações afetam o bem-estar entre as partes, sendo dificilmente observáveis pelo principal. Este tipo de relação coloca em cena o problema de assimetria de informações entre 0 agente e 0 principal (J ensen e Meckling, 1976; Eisenhardt, 1989), que beneficia o primeiro em detrimento do segundo.

A teoria analisa, então, como um ator econômico (principal) estabelece um sistema de compensação (contrato) que motive o outro ator (agente) a agir de acordo com 0 interesse do primeiro. A questão primordial é a dificuldade de monitorar o esforço dos atores econômicos envolvidos em uma transação, o que torna ainda mais complexa a elaboração dos contratos. Por esta razão, são incluídos esquemas de incentivos baseados na performance observada. Na elaboração de um esquema de incentivos, as partes envolvidas enfrentam o trade off entre incentivos ótimos e repartição de riscos ótima (J ensen \& Meckling, 1976).

Da análise dos contratos surgem dois conceitos básicos dessa teoria, a saber: a seleção adversa e o risco moral. A sel eção adversa deriva do custo de acesso à informação e consiste no fato de que a seleção do produto a ser demandado ocorre de forma ineficiente, portanto, adversa, em função da assimetria de informação entre os ofertantes e demandantes (Carvalho et alli, 2000). O exemplo clássico de seleção adversa existente na literatura é o do mercado de automóveis usados nos Estados Unidos (Akerlof, 1970). Já o risco moral se baseia nas ações dos agentes, que podem acabar influenciando esse risco após a transação ser efetivada (Carvalho et alli, 2000). Um exemplo de risco moral ocorre na indústria de seguros, onde diferentes consumidores que contrataram o seguro vão ter distintos comportamentos quanto ao cuidado com o bem segurado, fato que vai modificar a probabilidade de ocorrência de sinistro entre eles.

Segundo Perrow (1991) as organizações reconhecem que os custos de uma seleção ótima são altos, por isso os postos de trabalho são definidos tendo como parâmetro o padrão médio de comportamento dos trabalhadores. A seleção adversa e o risco moral seriam, portanto, problemas com os quais as organizações teriam que lidar diariamente. Observa também que a Teoria da Agência parece ser ideologicamente incapaz de tratar com igualdade ambas as partes contratantes, visto que não reconhece a assimetria do poder e de recursos dentro da organização. Além disso, a teoria não considera relevantes os aspectos cooperativos mais amplos da vida social.

Diante do exposto, o presente trabalho procura explorar os conflitos de agência existentes em dois diferentes tipos de organizações, a saber: empresas tradicionais e cooperativas de crédito.

Tal estudo se justifica pelo fato de que, segundo Cuevas e Fischer (2006), as cooperativas de crédito estão entre as instituições financeiras menos estudadas. Além disso, recentemente o cooperativismo de crédito no Brasil vem sofrendo mudanças normativas importantes. O objetivo principal dessas mudanças é aumentar a participação das cooperativas de crédito no Sistema Financeiro Nacional (SFN). 
No cerne dessas mudanças, está o apoio governamental para o desenvolvimento do setor cooperativista. 0 intuito é transformá-lo num importante alicerce para o aumento da oferta de crédito e da concorrência de serviços financeiros, proporcionando o maior benefício a classes sociais menos favorecidas.

No Relatório de Estabilidade Financeira do Banco Central do Brasil de maio de 2003, está confirmada a intenção de se criar avanços na regulamentação do setor cooperativista de crédito e viabilizar as metas do Governo de expansão e redução do custo do crédito no Brasil. De acordo com o documento, as recentes medidas regulamentadas para a constituição e funcionamento das cooperativas de crédito permitem assegurar, no médio prazo, significativo crescimento deste segmento no SFN.

O marco inicial das alterações normativas recentes é o início da vigência da Resolução CMN 3.106, de 25 de junho de 2003. Desde então, uma quantidade representativa de cooperativas de crédito aproveitaram as possibilidades implementadas por essa resolução, seja em virtude de constituição de novas cooperativas ou pela transformação de cooperativas até então existentes para a modalidade de livre admissão de associados.

0 estudo encontra-se organizado com a primeira seção, representada por esta introdução do assunto pesquisado, a seção dois, que aborda os principais conceitos e aspectos teóricos ao entendimento dos conflitos de agência em empresas tradicionais. A seção três apresenta os principais conceitos e aspectos teóricos ao entendimento dos conflitos de agência em cooperativas de crédito, tendo por base estudos já realizados sobre essas organizações. Finalmente, a seção quatro discute e apresenta considerações com enfoque comparativo sobre os principais aspectos dos conflitos de agência em cada tipo de organização em estudo.

\section{CONFLITOS DE AGÊNCIA EM EMPRESAS TRADICIONAIS}

Os conflitos de agência tornaram-se importantes a partir da evolução dos mercados de capital. De acordo com Segatto-Mendes (2001), a teoria da agência proporcionou uma análise das diferentes relações contratuais existentes no seio da empresa, incluindo o conceito de relação de agência, que normalmente envolve um agente principal (proprietário) que solicita a um agente (gestor) que atue por sua conta e em prol de seus interesses, sendo oferecido em troca dos serviços uma remuneração.

A Teoria da Agência relata de forma mais abrangente a possibilidade de divergência de interesses de acionistas e gestores, onde um tenta tirar proveito de vantagens advindas de uma posição privilegiada. Segundo o Código das Melhores Práticas do Instituto Brasileiro de Governança Corporativa (IBGC, 2003), ocorre conflito de interesses quando alguém não é independente em relação à matéria em discussão e mesmo assim pode influenciar ou tomar decisões motivadas por interesses distintos daqueles da sociedade. Segundo Andrade e Rossetti (2004), em virtude de estratégias defensivas, os dirigentes tendem a minimizar o nível da sua exposição, com proveito de maior nível de "desconcentração" dos acionistas (principal), o que repercute no nível de disponibilidade e profundidade das informações repassadas àqueles interessados.

A preocupação inerente ao ambiente empresarial, principalmente no caso em que sua propriedade é dispersa, é assegurar que as decisões dos agentes agreguem o maior valor possível às ações diante das estratégias e condutas possíveis de serem implementadas. Os mecanismos de controle devem então garantir que os gestores não 
tomem decisões conflitantes com o objetivo de maximizar o valor da empresa e, conseqüentemente, das ações.

De acordo com Hölmstrom (1979), os conflitos entre acionistas e gestores podem ser minimizados por meio de incentivos apropriados, que limitem o comportamento conflitante por parte dos agentes (gestores). O compartilhamento do capital surge como exemplo de incentivo, alinhando o interesse na valorização da ação. Nesse sentido, os princípios de governança corporativa, reconhecida como conjunto de mecanismos que alinham interesses de gestores e acionistas, passou a ser interpretada pelo mercado como aspecto positivo com relação ao retorno do acionista.

Para Louvet e Taramasco (2007), no relacionamento entre capitalista e gestores, a nomeação do dirigente baseia-se nas seguintes variáveis: proporção do capital detido pelo dirigente, taxas de distribuição dos lucros, taxa de retiradas pelos dirigentes e custos de indenização. Entretanto, por mais adequado que sejam os incentivos, sempre existem margens para ações discricionárias dos gestores (agentes), não sendo possível eliminá-las por completo.

Quanto ao aspecto da difusão da propriedade, para Andrade e Rossetti (2004), a menor necessidade de monitoramento por parte dos proprietários e a redução dos custos de agência acabam sendo vantagens no caso de maior concentração da propriedade, principalmente em situações de relevante dificuldade de monitoração das decisões gerenciais e de coordenação de obj etivos dos proprietários.

Além do conflito de interesses entre propriedade e gestão, podem ainda ser identificados outros dois tipos de conflitos de agência na estrutura das empresas, agora mais relacionados às estruturas de fonte de capital. Um tipo é o conflito entre acionistas (capital próprio) e credores (capital de terceiros). De acordo com Harris e Raviv (1991), em algumas empresas, essa categoria pode ser apontada como determinante de sua estrutura de capital, tendo com meta a redução de interesses potencialmente divergentes entre diversos grupos com direitos sobre os recursos da empresa.

Harris e Raviv (1991) definem que "conflicts batween debtholders and equityholders arise bacause the debt contract gives equityholders an incentive to invest suboptimall. ...the debt contract provides that if an investment yields large return, well above the face value of the debt, equityholders capture most of the gain. If, however, the investment fails, bacause of limited liability, debtholders bear the consequence."

Outro tipo de conflito potencial relacionado à estrutura de capital da empresa, e relevante na realidade do mercado de capitais brasileiro, é constituído pelas divergências de interesses entre acionistas minoritários e acionistas majoritários. Apesar de Jensen e Meckiling (1976) apontarem dois conflitos na empresa, credores e acionistas e gestores e acionistas, não se pode considerar que existe perfeita convergência de interesses entre acionistas controladores e minoritários. De forma ainda mais abrangente, a conduta dos gestores em relação à decisão de qual grupo de interesse deve ser defendida, minoritários ou controladores, pode criar novos níveis de conflito de interesses. Como exemplo pode ser citada a possibilidade de proteção pelo gerente dos interesses do grupo de controle, que possui o poder de elegê-lo e destituí-lo.

Segundo La Porta et al (1998) apud Soares e Kloeckner (2005), não obstante o fato da teoria de finanças se pautar pela propriedade pulverizada, a concentração acionária em todo o mundo é maior do que eles consideravam, com comum distinção dos acionistas em duas classes: minoritários e controladores. Soares e Kloeckner (2005) apontam para o fato de que esses atores possuem direitos e interesses diferentes.

É de amplo conhecimento que o investidor, ao entrar no mercado de capitais, busca a otimização de três aspectos básicos: retorno, prazo e proteção, e ao analisá-lo, realiza 
projeção de sua rentabilidade, liquidez e grau de risco. No mercado de capitais brasileiro, ocorrências já interferiram na rentabilidade do título investido e no grau de risco do investimento, referente à segurança advinda do conflito de interesses existente entre acionista controlador e acionista minoritário.

No contexto desses conflitos, pode ser atribuída grande importância aos Conselhos de Administração, evidenciado como o instrumento de controle dos conflitos de agência nas empresas de capital aberto, sendo não só o responsável pela monitoração do gestor, mas também como o meio viável de alinhar interesses na relação gestor $x$ capital e capital majoritário x capital minoritário. Em uma rápida análise de códigos de melhores práticas tais como, CVM, IBGC, OECD e BIS, pode ser observado esse papel de destaque.

Carver e Carver (2001) criticam a importância dada aos Conselhos de Administração e afirmam que eles podem funcionar de forma pouco efetiva, no caso de reunirem pessoas experientes individualmente, mas que, em grupo não exerçam as suas funções. No caso de divergências entre acionistas e companhia ou entre controladores e minoritários, a Comissão de Valores Mobiliários (CVM) recomenda que os conflitos de interesse sejam resolvidos por meio de uma instância superior de arbitragem, que visa acelerar a solução dos impasses.

Segundo Andrade e Rossetti (2004) com base nos fundamentos da Lei SarbanesOxley, as principais normas que orientam as ações dos gestores da empresa devem ser baseadas em quatro princípios:

- conformidade legal (compliance), em que executivos devem adotar um código de ética;

- prestação responsável de contas (accountability);

- transparência (disclosure); e

- senso de justiça (fairness).

\section{CONFLITOS DE AGÊNCIA EM COOPERATIVAS DE CRÉDITO}

Em relação às cooperativas de crédito, Cuevas e Fischer (2006) destacam que esse tipo de instituição financeira apresenta vantagens claras sobre as outras, mas que também possui fraquezas que não podem ser ignoradas. A mais importante dessas vantagens é a solução natural dos problemas da seleção adversa do mercado financeiro tradicional que resultam no racionamento do crédito. 0 mutualismo permite 0 acesso aos serviços financeiros para agentes (micro e pequenos empresários, pequenos agricultores e população mais pobre) que, caso contrário, teriam o seu crédito racionado.

Segundo Branch e Baker (2000), o principal problema de agência em sociedades cooperativas advém do fato de que, muitas vezes, não há uma separação clara entre os proprietários (associados) e os tomadores de decisão (gerentes) das cooperativas. Embora esse problema possa ocorrer em qualquer tipo de instituição financeira, para os autores, existem pelo menos quatro fatores complicadores no caso específico das cooperativas de crédito. O primeiro deles advém do fato de que os proprietários (associados) são simultaneamente seus clientes. 0 segundo surge porque seus clientes podem ser classificados em dois tipos com diferentes interesses, a saber: os clientes que são poupadores e os que são tomadores de empréstimos. 0 terceiro fator está relacionado com a atribuição, para a eleição da direção da cooperativa de crédito, de apenas um voto para cada associado sem levar em conta a quantia investida por cada um deles. Finalmente, na 
maioria das vezes, observa-se que os associados não possuem muita experiência empresarial ou mesmo grande conhecimento financeiro.

Para Cuevas e Fischer (2006), há dois conflitos principais de agência que dominam as cooperativas de crédito. São elas: o conflito tomador de empréstimo x poupador e 0 conflito associado x gerência. Os autores também afirmam que o conflito tomador de empréstimo $x$ poupador tem recebido relativamente pouca atenção por parte dos pesquisadores. Apesar disso, há uma forte evidência empírica que este conflito pode ser significativo e ter contribuído para muitos dos fracassos encontrados nas cooperativas de crédito latino americanas. Naquela região, um grande número de distorções contribuiu para criar um viés em direção aos tomadores de empréstimo, o que explicaria os fracassos citados. Dessa forma, seria fundamental proteger os interesses tanto dos poupadores quanto dos tomadores de empréstimos, impedindo que a direção seja controlada pelos interesses dos tomadores de empréstimo.

Em concordância com os autores anteriores, Westley e Branch (2000) observam que há uma tendência dos tomadores de empréstimo dominarem a direção da cooperativa de crédito e a administrarem de acordo com o interesse desse grupo. Esse fenômeno faz com que as pessoas procurem a associação à cooperativa de crédito com o intuito principal de tomar empréstimos mais baratos e não para depositar recursos, o que cria condições que potencializam o aumento da inadimplência.

Já Branch e Baker (2000) observam que as diferentes visões dos associados em relação à cooperativa gerarão diferentes formas de comportamento. Aqueles cooperados que se associarem com o objetivo de depositar suas economias focarão a segurança da instituição e o adequado nível de remuneração de seus depósitos, com preocupação a respeito dos custos das transações. Dessa forma, apoiarão também a melhoria de sua estrutura, o que, entre outros critérios, inclui a profissionalização da gerência. Por outro lado, os que se associam com o objetivo de obter empréstimos estabelecerão baixas taxas para os empréstimos e depósitos e terão um comportamento mais indulgente em relação à concessão do crédito e à inadimplência. Além disso, serão mais agressivos em relação à mobilização de depósitos e menos preocupados com a profissionalização da administração.

0 conflito associado $x$ gerência é uma importante fonte de vulnerabilidade na governança nas cooperativas de crédito e tem sido objeto de um grande número de estudos acadêmicos (Cuevas e Fischer, 2006). Para Westley e Branch (2000), a resolução desses problemas de agência depende de definições claras das regras que determinam as responsabilidades de cada um dos envolvidos na gestão da cooperativa. Essas definições deveriam constar explicitamente em seu Estatuto Social através da utilização dos seguintes princípios:

a) Definição clara das alçadas de decisão no âmbito da gerência da cooperativa de crédito para de limitar o envolvimento da diretoria nas operações do dia a dia a fim de separar claramente a tomada da decisão de sua execução;

b) Definição de critérios técnicos mínimos para que um associado possa ser eleito para os órgãos de administração da cooperativa de crédito, impedindo, dessa forma, que pessoas não qualificadas sejam eleitas;

c) Definição das atribuições do comitê de supervisão (auditoria interna) para que este possa funcionar sem a interferência da gerência da cooperativa de crédito e seja responsável pela verificação da aderência (compliance) em relação ao Estatuto Social, ao controles internos e a atuação da gerência;

d) Definição da política e critérios utilizados na concessão do crédito com a identificação dos responsáveis pela análise de crédito que deverá ser feita a partir de critérios técnicos; 
e) Definição clara da responsabilidade da direção em relação às operações e ao funcionamento da cooperativa de crédito em geral, bem como as punições em caso do não cumprimento. Dessa forma, deveriam ser previstos, inclusive, mecanismos para substituição de dirigentes que não cumpram suas obrigações;

f) Definição de códigos de ética e de controles dos empréstimos realizados para membros da administração a fim de evitar conflitos de interesse;

g) Previsão de rotação de cargos entre os membros da administração. Uma limitação de dois ou três mandatos para cada dirigente permitiria 0 aparecimento de novas idéias, evitando a dominação da administração por um pequeno grupo por períodos de tempo extensos.

Segundo Cuevas e Fischer (2006), uma das vertentes mais importantes no estudo do conflito associado $x$ gerência foca suas atenções no efeito de separar a propriedade (associados) dos controles (gerência), visto que os interesses de ambos divergem em alguns pontos básicos. A relevância no controle desse conflito se justifica pelo fato das decisões dos gerentes em relação à aplicação dos recursos disponíveis é a principal fonte de fracasso das cooperativas de crédito, 0 que deveria ser um foco central das regulamentações prudenciais e da supervisão externa.

\section{CONSIDERAÇÕES FINAIS}

A fim de comparar os problemas de agência descritos nos dois tipos de organização estudados, foi elaborada a seguinte tabela comparativa com o objetivo de focar as semelhanças e diferenças encontradas. 
Tabela I: Conflitos de Agência

\begin{tabular}{|c|c|c|}
\hline Conflito de Agência & Empresas Tradicionais & Cooperativas de Crédito \\
\hline $\begin{array}{l}\text { Credor } \\
\text { X } \\
\text { Acionista }\end{array}$ & $\begin{array}{l}\text { Determinante da estrutura de } \\
\text { capital, pois define a combinação } \\
\text { de interesses entre diversos } \\
\text { grupos que fornecem recursos e } \\
\text { possuem direitos sobre o fluxo de } \\
\text { caixa gerado pela empresa. }\end{array}$ & $\begin{array}{l}\text { Não aplicável, considerando } \\
\text { que os fornecedores de } \\
\text { recursos são os próprios } \\
\text { associados, que participam } \\
\text { tanto da formação das quotas } \\
\text { de patrimônio líquido como } \\
\text { dos saldos depositados. }\end{array}$ \\
\hline $\begin{array}{c}\text { Acionista minoritário } \\
X \\
\text { Acionista majoritário }\end{array}$ & $\begin{array}{l}\text { Ligado ao aspecto de difusão da } \\
\text { propriedade, } \\
\text { dificuldades de moporciona } \\
\text { relatadas por Andrade e Rossetti } \\
\text { aos detentores das parcelas } \\
\text { difusas, mantendo uma parcela } \\
\text { concentrada que facilita a } \\
\text { condução das atividades e a } \\
\text { influência sobre o administrador } \\
\text { de acordo com interesses } \\
\text { próprios. }\end{array}$ & $\begin{array}{l}\text { Não aplicável, sociedade de } \\
\text { pessoas, onde os associados } \\
\text { possuem poder de deliberação } \\
\text { (votos) equivalentes e } \\
\text { independe do percentual de } \\
\text { participação no capital. }\end{array}$ \\
\hline $\begin{array}{c}\text { Associado/acionista } \\
\text { X } \\
\text { gerência }\end{array}$ & 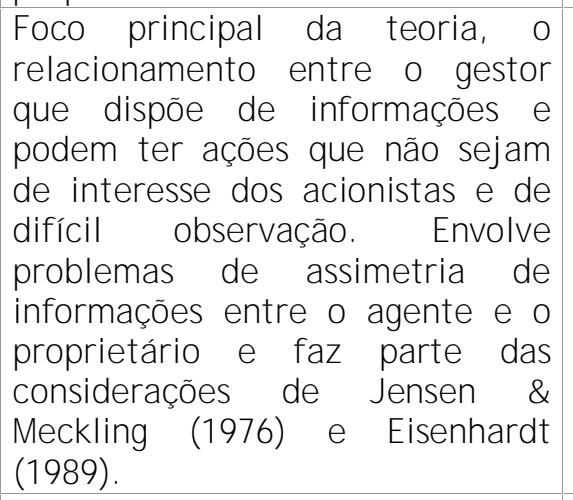 & $\begin{array}{l}\text { Relevante na realidade de } \\
\text { delegação de poderes. Estudos } \\
\text { empíricos sugerem que esta é } \\
\text { a principal fonte de fracasso } \\
\text { das cooperativas de crédito, o } \\
\text { que demonstra a necessidade } \\
\text { de regulamentação prudencial } \\
\text { específica para essas } \\
\text { instituições. }\end{array}$ \\
\hline $\begin{array}{c}\text { Tomador de empréstimo } \\
X \\
\text { poupador }\end{array}$ & $\begin{array}{l}\text { Apesar dos depositantes e } \\
\text { devedores tentarem ter as } \\
\text { melhores margens em seu } \\
\text { benefício, esses interesses não } \\
\text { chegam a caracterizar um conflito } \\
\text { em interesses de agentes na } \\
\text { condução e administração de } \\
\text { instituições } \\
\text { tradicionais. }\end{array}$ & $\begin{array}{l}\text { Relevante, pois ambos os } \\
\text { grupos exercem pressão sobre } \\
\text { a conduta dos gestores, com a } \\
\text { possibilidade de concessão de } \\
\text { empréstimos subsidiados e/ ou, } \\
\text { possivelmente, remuneração } \\
\text { de depósitos acima da média } \\
\text { do mercado. Pode levar à falta } \\
\text { de competitividade, elevação } \\
\text { no risco de crédito e fracasso } \\
\text { da cooperativa. }\end{array}$ \\
\hline
\end{tabular}

Fonte: elaboração própria

Pode-se observar que muitos conflitos de agência observados nas cooperativas de crédito são diferentes dos existentes nas empresas tradicionais. Isso poderia ser considerado um indicativo de que podem ser necessárias soluções de governança diferentes para este primeiro tipo de organização.

No entanto, segundo Branch e Baker (2000), os conflitos de agência nas cooperativas de crédito podem ser superados desde que sejam adotadas providências, tais como regras de governança bem definidas, controles internos eficazes, adequação dos serviços prestados, adoção de regulamentações prudenciais e supervisão externa. Cabe ressaltar que as providências citadas vão ao encontro do que também é preconizado para 
as empresas tradicionais, o que, a princípio, poderia ser considerada uma contradição caso se leve em conta a existência de conflitos de agência diferentes.

Diante do exposto, sugerimos que os novos estudos de governança em cooperativas de crédito, principalmente aqueles que tenham o objetivo de pesquisar sobre melhores práticas a serem adotadas nestas instituições, devam ter como base e referencial os conflitos de agência específicos deste tipo de organização. Tal fato poderá levar a determinação de práticas mais apropriadas e não somente aos mecanismos tradicionalmente descritos como panacéia para resolução de problemas de governança corporativa.

\section{REFERÊNCIAS}

ANDRADE, Adriana; ROSSETTI, J osé Paschoal. Governança corporativa: fundamentos, desenvolvimento e tendências. São Paulo: Atlas, 2004.

AKERLOF, George A. The Market for 'Lemons': Quality Uncertainty and the Market Mechanism. The Quarterly Journal of Economics, MIT Press, vol. 84(3), pages 488-500, 1970.

BANCO CENTRAL DO BRASIL (BACEN). Relatório de estabilidade financeira. Brasília: Banco Central do Brasil, Maio 2003.

BANK FOR INTERNATIONAL SETTLEMENTS (BIS). Aperfeiçoando a governança corporativa em organizações bancárias. Basiléia, 2006

BOLSA DE VALORES DE SÃO PAULO (BOVESPA). Regulamento de práticas diferenciadas de governança corporativa nível 1. São Paulo, 2006.

BOLSA DE VALORES DE SÃO PAULO (BOVESPA). Regulamento de práticas diferenciadas de governança corporativa nível 2. São Paulo, 2006.

BRANCH, Brian, BAKER, Christopher. Chapter 11: Overcoming Credit Union Governance Problems. IN WESTLEY, Glenn D., BRANCH, Brian (org.). Safe money: building effective credit unions in Latin America. Washington: Inter-American Development Bank and World Council of Credit Unions, 2000.

CARVALHO, Fernando J. Cardim de, SOUZA, Francisco Eduardo Pires de, SICSÚ, João, PAULA, Luiz Fernando Rodrigues de, STUDART, Rogério. Economia Monetária e Financeira - Teoria e Prática. Rio de J aneiro: Campus, 2002.

CARVER, John; CARVER, Miriam. Carver's policy governance model in nonprofit organizations. Gouvernance: Revue Internationale, Hiver, Vol. 2, n. 1, p. 30-48, 2001. Disponível em: http:// www. carvergovernance.com/pg-np.htm. Acesso em: 12 set. 2006.

CONSELHO MONETÁRIO NACIONAL (CMN). Resolução 3.106, de 25 de junho de 2003. Dispõe sobre requisitos e procedimentos para a constituição, a autorização para funcionamento e alterações estatutárias, bem como para o cancelamento da autorização para funcionamento de cooperativas de crédito. Diário Oficial da União, Brasília: 25.06. 2003.

CONSELHO MONETÁRIO NACIONAL (CMN). Resolução 3.321, de 30 de setembro de 2005. Dispõe sobre a constituição, a autorização para funcionamento, alterações estatutárias e 0 cancelamento de autorização de cooperativa de crédito e sobre realização de auditoria externa em cooperativa singular de crédito. Diário Oficial da União, Brasília: 30.09. 2005 
CUEVAS, Carlos E., FISCHER, Klaus p. Cooperative financial institutions: issues in governance, regulation, and supervision. Washington: The World Bank, 2006.

EISENHARDT, K. M. Agency theory: an assessment and review. Academy of Management Review, 1989, v. 15, n. 1, 57-74.

HARRIS, M., RAVIV, A. The Theory of Capital Structure. The J ournal of Finance, Vol. 46, No. 1. (Mar., 1991), pp. 297-355.

HÖLMSTROM, Bengt. Moral Hazard and Observability. The Bell J ournal of Economics, Vol. 10, No. 1. (Spring, 1979), pp. 74-91.

INSTITUTO BRASILEIRO DE GOVERNANÇA CORPORATIVA (IBGC). Código das Melhores Práticas de Governança Corporativa. São Paulo, 2003.

J ENSEN, M., MECKLING, W. Theory of the firm: Managerial behavior, agency costs, and ownership structure. J ournal of Financial Economics, 3, 305-360, 1976.

LOUVET, Pascal, TARAMASCO, Ollivier, "Stock-Based Pay: An Incentive for Performance or a Compensation for Competence? How to Compensate a Manager When He is Competent?" (November 7, 2007). Available at SSRN: http:// ssrn. com/ abstract $=1028250$

ORGANIZAÇÃO PARA A COOPERAÇÃO E DESENVOLVIMENTO ECONÔMICO (OCDE). Princípios da OCDE sobre governança corporativa. Paris, 2004

PERROW, C. Sociologia de las Organizaciones. España: McGraw-Hill, 1991.

ROSS, S. The economic theory of agency: the principal's problem. American Economic Review, 20 (2), 22-32, 1973.

SEGATTO-MENDES, A. P. Teoria de agência aplicada à análise de relações entre os participantes dos processos de cooperação tecnológica universidade-empresa. Departamento de Administração da Universidade Federal de São Paulo, 2001 (Tese de Doutorado).

SOARES, Rodrigo Oliveira ; KLOECKNER, G. O. Controle acionário definido e endividamento: Uma abordagem multi-método. In: $V$ Congresso USP Controladoria e Contabilidade, 2005, São Paulo. Anais do V Congresso USP Controladoria e Contabilidade, 2005. v. 1. p. 1-15.

WESTLEY, Glenn D., BRANCH, Brian (org.). Safe money: building effective credit unions in Latin America. Washington: Inter-American Development Bank and World Council of Credit Unions, 2000.

\title{
ENDEREÇO DOS AUTORES
}

\author{
Universidade Federal de Minas Gerais \\ Faculdade de Ciências Econômicas \\ Departamento de Ciências Administrativas \\ Av. Antônio Carlos, 6627 - Prédio FACE - Bloco 01 - 4 andar - Sala 4030 \\ Pampulha \\ Belo Horizonte, MG - Brasil \\ 31270-901
}

